pair examined 14 sequencing papers published last year in Science, Nature and Nature Genetics, and found that the publications often lacked essential details needed to reproduce the analysis - the papers referenced merely bioinformatics software, for example, without noting the version used or the value of key parameters.

The two researchers presented their findings at the Advances in Genome Biology and Technology meeting in Marco Island, Florida, on 2 February. Although their account has not been published, it does not seem to have surprised anyone in the field. Indeed, it builds on a 2009 paper in Nature Genetics that found similar omissions in published accounts of microarray experiments. (J. P A. Ioannidis et al. Nature Genet. 41, 149-155; 2009). In this case, findings from 10 of the 18 studies analysed could not be reproduced, probably because of missing information.

If genomics were as politicized as climate science, the authors of studies in which the information trail is missing would probably face catcalls, conspiracy charges and demands for greater transparency and openness. Instead, many in the field merely shrug their shoulders and insist that is how things are done. Bioinformatics is a fast-paced science in which software and standards for data analysis change rapidly and with them, the protocols and workflows of users.

Nature does not require authors to make code available, but we do expect a description detailed enough to allow others to write their own code to do a similar analysis.

Some in the field say that it should be enough to publish only the original data and final results, without providing detailed accounts of the steps in between. Others argue that it is pointless to document the version of the software used, as new incarnations of programs differ little. But that is not always the case. Edward McCabe, then at the California NanoSystems Institute at the University of California, Los Angeles, was so perturbed when different versions of the

same bioinformatics software gave wildly different results that he published a paper on it (N. K. Henderson-Maclennan et al. Mol. Genet. Metab. 101, 134-140; 2010). Reviewers resisted its publication, asking what was new about the findings, as it was already common knowledge that different software versions could dramatically affect analyses. There is a troubling undercurrent here: that the problem lies not with the lack of information, but rather with those who find the incomplete information a problem, such as researchers who are new to the field.

Transparency is a laudable goal, but given the complexity of the analyses, is it realistic? There are certainly examples of stellar documentation. The 1000 Genomes Project, for example, a project to

"Transparency is a laudable goal, but given the complexity of the analyses, is it realistic?" vidual scientists, who have more flexible and varied research goals. Nevertheless, tools are coming online to simplify documentation of the complex analyses required for genome analysis. These include freely available programs such as Taverna (www.taverna.org.uk) and Nekrutenko's more user-friendly Galaxy (http://main.g2.bx.psu.edu). Neither of these is perfect, but they illustrate the level of detail that could enrich published reports.

As genome sequencing spreads from the large, centralized sequencing centres that largely pioneered the technique into smaller labs and clinics, it is important that the community consider such solutions.

\section{Line in the sand}

\section{In the wake of the revolution, Egypt should embrace a future based on scientific thinking.}

$\mathrm{O}$ n Saturday, Wael Ghonim, a Google executive who helped to instigate Egypt's revolution and has become its reluctant icon, tweeted, "Go back to your work on Sunday, work like never before and help Egypt become a developed country."

More than two weeks of nationwide protests have finally toppled Hosni Mubarak, president of the country and leader of its repressive regime since 1981, who handing power to Egypt's Supreme Military Council in the early evening of 11 February. Considerable political uncertainty remains, but activists and intellectuals are vigilant, and insist that they will protect their revolution. This was a peaceful uprising, in which groups of educated youth attracted millions onto the streets to call for democracy, freedom and change: a movement with an uplifting sense of civic responsibility. For the first time, the faces of ordinary young Egyptians - and their aspirations for fundamental civil liberties and self-determination - could be seen internationally. So, what happens now, and what will it mean for science's role in the development of the Arab world's most populous nation?

Egypt's intellectuals and youth largely share Ghonim's instinct that their most urgent task is to begin a radical reconstruction of the country's society and economy. They know that an overhaul of the education and science systems will be crucial to this restructuring, unleashing the bottled-up energy and creativity of a youth that has been stifled for too long. Leading Egyptian researchers - including Ahmed Zewail, a chemist at the California Institute of Technology in Pasadena, who won the 1999 Nobel Prize in Chemistry and is

currently in Egypt to lend his hand to efforts to transition to democracy - are right to argue that education and research must be a top priority. They will be vital to taking the new Egypt and its young workforce into the twenty-first century, and to creating a more enlightened and productive society.

Research in Egypt, as in much of the Arab world, has long lagged behind that elsewhere, receiving only a paltry $0.2-0.3 \%$ of gross domestic product. Mubarak's regime was preoccupied instead with geopolitical security - an interest shared by the United States and other Western powers that propped it up - and neglected to invest in the infrastructure necessary for an educated and creative workforce. This contributed to the accumulation of social and economic woes that helped the revolution to win popular support.

As in Tunisia, the scene of an equally successful uprising this year, Egypt's repressive regime stifled research initiatives, and placed its cronies, rather than top scientists, in positions of academic power. Frustrated scientists left to seek opportunities abroad. Education in the country suffers similar problems: it is often based on rote learning, which smothers debate, creative thinking and entrepreneurship. As one Egyptian researcher told Nature, the universities "are simply assembly lines that produce thousands of unskilled graduates every year".

With sufficient investment, realistic plans can be drawn up to reform and revamp Egypt's education and research systems. Vast expertise is available both inside and outside the country. But that alone will not be enough: democracy, freedom and a new culture that encourages criticism and creativity must underpin any reforms. As Ismail Serageldin, director of the Library of Alexandria, put it to

D NATURE.COM To comment online, click on Editorials at: go.nature.com/xhunqu
Nature on the eve of Mubarak's fall: "Building science is not just a question of money and projects, it is also about a whole climate of research, of freedom of enquiry, freedom of expression, education, the ability to question." - 